\title{
Competing RNA secondary structures are required for mutually exclusive splicing of the Dscam exon 6 cluster
}

\author{
GEMMA E. MAY, SARA OLSON, C. JOEL MCMANUS, and BRENTON R. GRAVELEY \\ Department of Genetics and Developmental Biology, University of Connecticut Stem Cell Institute, University of Connecticut Health Center, \\ Farmington, Connecticut 06030, USA
}

\begin{abstract}
Alternative splicing of eukaryotic pre-mRNAs is an important mechanism for generating proteome diversity and regulating gene expression. The Drosophila melanogaster Down Syndrome Cell Adhesion Molecule (Dscam) gene is an extreme example of mutually exclusive splicing. Dscam contains 95 alternatively spliced exons that potentially encode 38,016 distinct mRNA and protein isoforms. We previously identified two sets of conserved sequence elements, the docking site and selector sequences in the Dscam exon 6 cluster, which contains 48 mutually exclusive exons. These elements were proposed to engage in competing RNA secondary structures required for mutually exclusive splicing, though this model has not yet been experimentally tested. Here we describe a new system that allowed us to demonstrate that the docking site and selector sequences are indeed required for exon 6 mutually exclusive splicing and that the strength of these RNA structures determines the frequency of exon 6 inclusion. We also show that the function of the docking site has been conserved for $\sim 500$ million years of evolution. This work demonstrates that conserved intronic sequences play a functional role in mutually exclusive splicing of the Dscam exon 6 cluster.
\end{abstract}

Keywords: alternative splicing; RNA structure; bioinformatics; recombineering; sequencing

\section{INTRODUCTION}

The vast majority of eukaryotic genes contain introns that are removed by pre-messenger RNA (pre-mRNA) splicing. In humans, $\sim 95 \%$ of genes encodes alternatively spliced mRNAs (Pan et al. 2008; Wang et al. 2008). Given the importance of alternative splicing in both regulating gene expression and in enhancing the diversity of the proteome (Nilsen and Graveley 2010), it is essential to understand how alternative splicing is regulated. Alternative splicing is regulated by many cis-acting sequences and trans-acting protein factors (Chen and Manley 2009; Nilsen and Graveley 2010). In addition, pre-mRNA structures are also important for alternative splicing regulation (Grover et al. 1999; Xing and Lee 2006). However, the role of RNA structure in alternative splicing is critically understudied (Xing and Lee 2006).

Reprint requests to: Brenton R. Graveley, Department of Genetics and Developmental Biology, University of Connecticut Stem Cell Institute, University of Connecticut Health Center, 400 Farmington Avenue, Farmington, CT 06030, USA; e-mail: graveley@neuron.uchc.edu; fax: (860) 679-8345.

Article published online ahead of print. Article and publication date are at http://www.rnajournal.org/cgi/doi/10.1261/rna.2521311.
Although the majority of alternatively spliced genes encode only a few different isoforms, many genes exist that encode tens, hundreds, and even thousands of distinct mRNAs (Nilsen and Graveley 2010). The Drosophila melanogaster Down Syndrome Cell Adhesion Molecule (Dscam) gene is the most extensively alternatively spliced gene known (Schmucker et al. 2000). Dscam contains 115 exons, 95 of which are alternatively spliced. The alternative exons are organized into four distinct clusters-the exon 4, 6, 9, and 17 clusters - that contain 12, 48, 33, and 2 variable exons each. Importantly, the exons within each cluster are alternatively spliced in a mutually exclusive manner. As a result, Dscam potentially encodes 38,016 different isoforms.

Multiple conserved sequences were previously identified in the introns of the exon 6 cluster (Graveley 2005; Anastassiou et al. 2006). Sequences upstream of each exon 6 variant (selector sequences) exhibit conserved base-pairing potentials with a docking site downstream of exon 5. As only one selector sequence at a time can interact with the docking site, we proposed a model in which competing RNA base-pairing interactions between the selector sequences and the docking site play a role in mutually exclusive splicing. We subsequently demonstrated that the protein HRP36 is required to repress splicing of the exon 6 variants and therefore also 
plays an important role in the mutually exclusive mechanism (Olson et al. 2007). Although the docking site-selector sequence interactions are strongly supported by their evolutionary conservation and compensatory changes, the function of these elements has not been experimentally tested.

Here, we have developed a BAC transfection system that facilitates the functional dissection of the docking site and selector sequences in the Dscam exon 6 cluster. We find that the docking site is required for efficient exon 6 splicing and that this function is evolutionarily conserved. We also show that the strength of the docking site and selector sequence interaction positively influences Dscam exon 6 variant selection. These results provide new insight into the mechanism of mutually exclusive splicing and exon choice in the Dscam exon 6 cluster.

\section{RESULTS}

\section{A system to study cis-elements involved in Dscam alternative splicing}

The Dscam exon 6 cluster is difficult to clone and manipulate because it is large (13 kilobase pairs [kbp]) and highly repetitive. These technical challenges have thus far prevented experiments that address the function of the docking site and selector sequences. To circumvent difficulties due to the size of the exon 6 cluster, we attempted to assay splicing using a "minigene" in which several of the exon 6 variants were removed. However, upon transfection into Drosophila S2 cells, these minigenes did not splice efficiently and did not recapitulate the splicing pattern of the endogenous gene (data not shown). Moreover, given the interplay between the promoter, transcription, and chromatin with splicing (Kornblihtt 2007), as well as potential polar effects, in which the splicing of exons in one portion of a pre-mRNA may influence splicing in other regions (Fededa et al. 2005), we felt it critical to use an experimental system that includes the entire Dscam gene and expression was driven by the endogenous Dscam promoter.

We developed a robust system to study RNA sequences that control Dscam alternative splicing using a $102 \mathrm{kbp}$ BAC containing the entire $62 \mathrm{kbp}$ Dscam gene (Venken et al. 2006) expressed from its endogenous promoter. To differentiate transcripts derived from the BAC and the endogenous Dscam gene, recombineering (Copeland et al. 2001) was used to replace sequences in constitutive exons 5 and 7 with the corresponding sequences from Drosophila virilis $\left(\right.$ Dscam $\left.^{5 / 7 v i r}\right)$. BACs containing the Dscam gene were transfected into S2 cells, total RNA was isolated, and splicing was assayed by RT-PCR. When using D. melanogaster primers, RT-PCR products were obtained in both Dscam $^{5 / 7 v i r}$ transfected cells (Fig. 1A, lane 1) and the untransfected control (Fig. 1A, lane 2). However, RT-PCR using D. virilis primers amplified a product from Dscam $^{5 / 7 v i r}$ transfected cells (Fig. 1A, lane 3), but not from the untransfected control (Fig. 1A,
A

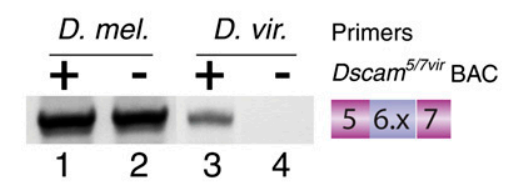

B

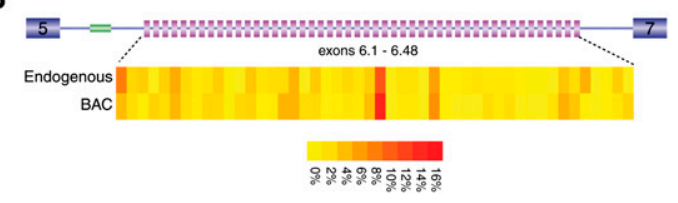

FIGURE 1. The Dscam BAC system. (A) D. melanogaster S2 cells were either untreated $(-)$ or transfected $(+)$ with a BAC containing the Dscam gene (Dscam ${ }^{5 / 7 v i r}$ BAC). RT-PCR was performed using exon 5 and 7 primers for either D. melanogaster or D. virilis. (B) The RT-PCR products were excised and sequenced using the multiplex sequencing method and the percent inclusion of each exon was calculated and represented as a heat map.

lane 4). These data indicate that the BAC transfection is efficient, and that $D s c a m^{5 / 7 v i r}$ transcripts can be specifically amplified in the presence of the endogenous Dscam gene.

To assess the biological relevance of our assay, we compared the relative frequency of exon 6 variant inclusion between transcripts derived from $\mathrm{Dscam}^{5 / 7 v i r}$ and the endogenous Dscam gene. We used a multiplexed, high-throughput sequencing assay that was recently developed (S Olson and BR Graveley, unpubl.) to accurately and quantitatively assay exon 6 variant usage. Briefly, S2 cells were transfected with the Dscam ${ }^{5 / 7 v i r}$ BAC in triplicate and RT-PCR was used to amplify transcripts containing the spliced exon 6 variant from the Dscam ${ }^{5 / 7 v i r}$ BAC and the endogenous gene separately. Custom, barcoded sequencing libraries were prepared from the resulting cDNA products and analyzed using multiplex high-throughput sequencing. High-throughput sequencing of cDNA products showed that this assay is repeatable as biological replicates were highly correlated (Pearson's $r$ ranged from 0.98849 to $0.99874[P<2.21 \times$ $10^{-39}$ ]) (Supplemental Table 1). Moreover, the frequency of exon 6 variant use averaged among replicates was highly correlated $\left(r=0.80, P=5.76 \times 10^{-12}\right)$ between BAC and endogenous Dscam transcripts (Fig. 1B). These data indicate that splicing of the transfected Dscam ${ }^{5 / 7 v i r}$ BAC accurately recapitulates splicing of the endogenous Dscam gene.

\section{The docking site is required for efficient exon 6 inclusion}

Having established this assay system, we tested whether the docking site was required for exon 6 inclusion. To do this, we deleted the docking sequence from the Dscam $^{5 / 7 v i r}$ BAC $\left(\right.$ Dscam $^{\Delta \text { Dock }}$ ) (Fig. 2A). After transfecting the modified BAC into S2 cells, exon 6 splicing was assayed by RT-PCR (Fig. 2B). Control RT-PCR reactions that amplified exons 
A

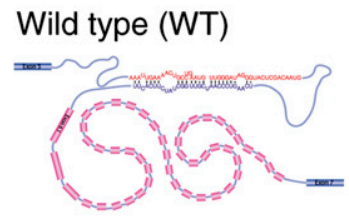

$\Delta$ Left

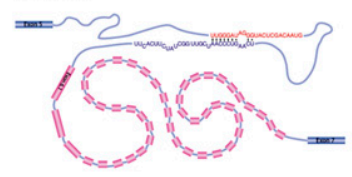

$\Delta$ Dock

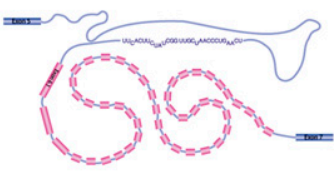

$\Delta$ Right

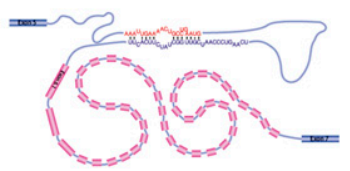

B

WT $\Delta$ Dock $\Delta$ Left $\Delta$ Right
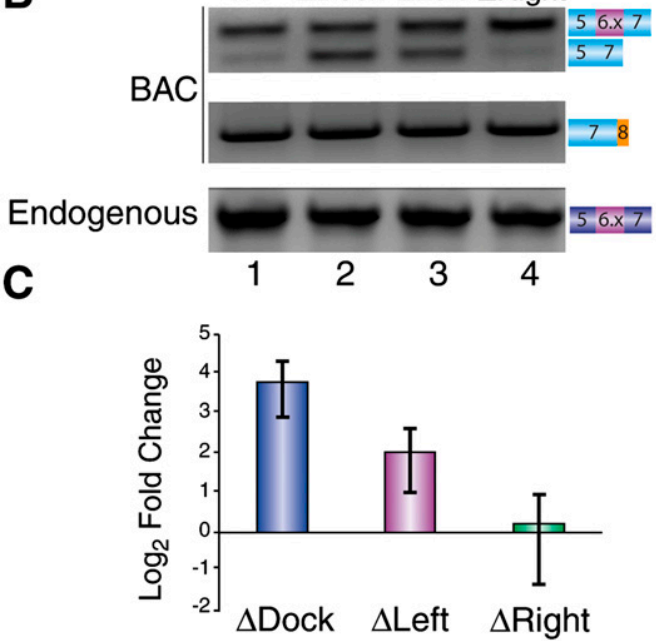

FIGURE 2. Dscam docking site mutations. (A) The $D_{\text {scam }}{ }^{5 / 7 v i r}$ BAC docking site (in red) was either completely deleted ( $\Delta$ Dock), the $5^{\prime}$ side was deleted ( $\Delta$ Left), or the $3^{\prime}$ side was deleted ( $\Delta$ Right). (B) D. melanogaster S2 cells were transfected with Dscam $^{5 / 7 v i r}$ BAC docking site mutant constructs. RT-PCR was performed with primers that anneal to BAC exons 5 and 7 (blue), BAC exon 7 and the junction of exons 7 and 8 (orange), or endogenous exons 5 and 7 (purple). When Dscam exon 6 was amplified, two products were observed, one which includes an exon 6 variant (6.x) and one lacking an exon 6 variant. (C) Dscam exon 6 skipping was measured for each mutant docking site BAC construct. Quantitative RT-PCR was used to measure skipping compared to the unaltered (WT) Dscam $^{5 / 7 v i r}$ BAC. The $\log _{2}$ fold change compared to WT is plotted for each mutant BAC construct with positive and negative error bars.

7 and 8 from the BAC using a species-specific exon 7 primer demonstrate that equal amounts of RNA was expressed from each construct (Fig. 2B). Interestingly, deletion of the docking site resulted in an increase in exon 6 skipping compared to the wild-type BAC (Fig. 2B, lanes 1,2). We developed an assay using quantitative RT-PCR to more accurately measure the amount of exon 6 skipped product in the docking site mutants. The amount of exon 5 spliced to exon 7 product (5-7) compared to a control sample, wildtype (WT) Dscam ${ }^{5 / 7 v i r}$ BAC was measured and the relative fold change of skipping compared to WT was calculated. Compared to WT, there was $14 \pm 6.4$-fold more exon 6 skipping when the docking sequence was deleted (Fig. 2C).
These results demonstrate that the docking site is required for efficient exon 6 inclusion.

In order to further dissect the docking site, the left and right portions of the docking site were deleted from the Dscam ${ }^{5 / 7 v i r}$ BAC (Fig. 2A). We again transfected these BACs into S2 cells and assayed exon 6 skipping using RT-PCR (Fig. 2B, lanes 3,4) and qRT-PCR (Fig. 2C). When the left side of the docking site was deleted (Dscam ${ }^{\Delta \text { Left }}$ ) we observed a $4.2 \pm 2.1$-fold increase in exon 6 skipping compared to WT (Fig. 2B, lane 3; Fig. 2C). However, deletion of the right side of the docking site $\left(\right.$ Dscam $\left.^{\Delta \text { Right }}\right)$ had little impact on exon 6 skipping (1.2 \pm 0.8 -fold) (Fig. 2B, lane 4; Fig. 2C). These results demonstrate that deleting the left side of the docking site has a stronger impact on exon 6 inclusion than deleting the right side.

We next tested whether the function of the docking site is conserved in other species. To do this, we used the Dscam gene from Daphnia pulex, a crustacean that is nearly 500 million years diverged from $D$. melanogaster. Despite this divergence, the $D$. pulex Dscam gene is remarkably similar to that of D. melanogaster, as it contains $7,26,17$, and 2 mutually exclusive exons in the same orientation and organization as D. melanogaster (Fig. 3A; Brites et al. 2008; Lee et al. 2009). Moreover, the core sequence of the docking site is highly conserved between $D$. pulex and D. melanogaster (Fig. 3B). To test if the function of the docking site is conserved in $D$. pulex, we constructed a minigene of the $D$. pulex exon 6 cluster and transfected it into D. melanogaster S2 cells. RT-PCR was performed using primers specific to constitutive exons 5 and 7 of D. pulex to assay exon 6 skipping. From the wild-type $D$. pulex minigene, we observed RT-PCR products corresponding to both exon 6 inclusion and skipping, though the skipping product was more abundant (Fig. 3C, lane 1). Nonetheless, when the docking site was deleted from the D. pulex minigene, exon 6 skipping was increased (Fig. 3C, lane 2). These results indicate that the $D$. pulex docking site is required for efficient exon 6 inclusion and that the function of the docking site is evolutionarily conserved.

\section{The docking site is required for determining exon 6 inclusion frequency}

To determine if the docking site plays a role in exon 6 variant usage, we assayed relative exon 6 use in the exon 6 containing RT-PCR products using high-throughput sequencing. When the docking site was completely removed ( $\Delta$ Dock), the vast majority $(68 \%)$ of transcripts used the first exon (6.1) (Fig. 4). Compared to the WT Dscam ${ }^{5 / 7 v i r}$ $\mathrm{BAC}$, there was a dramatic decrease in the inclusion frequency of all but a few exons. Interestingly, the relative use of the exons close to constitutive exon 7 (exons 6.406.48) did not significantly decrease. In fact, two of these exons, 6.45 and 6.46, actually increased in frequency compared to the wild-type Dscam ${ }^{5 / 7 v i r}$ BAC. A very similar 
A

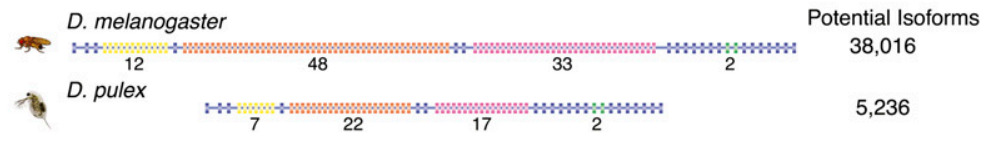

B D. melanogaster
D. pulex

C

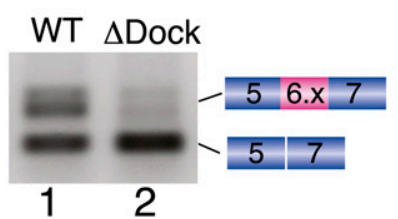

FIGURE 3. The Daphnia pulex Dscam docking site is functionally conserved. (A) D. melanogaster and D. pulex Dscam gene organization. (B) The docking site sequences from $D$. melanogaster and D. pulex are shown. The conserved sequence is highlighted in orange. $(C)$ D. melanogaster S2 cells were transfected with the D. pulex Dscam minigene (WT) and a minigene in which the docking site was deleted ( $\triangle$ Dock). RT-PCR was performed with primers that anneal to D. pulex exons 5 and 7 . Two products were observed, one that includes an exon 6 variant (6.x) and one that lacks an exon 6 variant.

pattern of exon 6 usage was observed for the Dscam ${ }^{\Delta L e f t}$; $41 \%$ of transcripts used exon 6.1 and exons close to constitutive exon 7 (exons 6.43, 6.45, and 6.46) increased in frequency. Interestingly, exons in the middle of the exon 6 cluster (exons 6.22, 6.23, and 6.24) also increased in frequency in Dscam ${ }^{\text {Left }}$ compared to the wild-type Dscam ${ }^{5 / 7 v i r}$ BAC. Although deleting the right side of the dock (Dscam ${ }^{\Delta \text { Right }}$ ) did not cause exon 6 skipping (Fig. 2), it resulted in a striking change in the relative use of most of the exon 6 variants (Fig. 4). As with the other docking site deletions, the frequency of exon 6.1 inclusion did increase to $12 \%$, exons $6.25,6.42$, and 6.43 were all included more frequently (Fig. 4). Taken together, these results show that the docking site is required for efficient exon 6 inclusion and plays a role in determining the frequency of each exon 6 variant.

\section{The strength of docking site and selector sequence interaction is correlated with exon 6 selection}

Due to the fact that each docking site-selector sequence secondary structure is different (Graveley 2005), the strength of these interactions is likely to vary. We hypothesized that the strength of these interactions could determine the efficiency of exon 6 use-stronger base-paring interactions would result in a higher frequency of inclusion of the downstream exon 6 variant, while weaker interactions would result in less inclusion. To test if the predicted pairing energy was correlated to the frequency of inclusion, we calculated the free energy $(\Delta G)$ of each of the predicted docking siteselector sequence interactions (Supplemental Table 3). We then calculated the Pearson's correlation between $\Delta G$ and the percent inclusion of each exon 6 variant from the endogenous Dscam gene. There was a small $(r=-0.16)$ but insignificant $(P=0.2616)$ correlation between the strength of docking site and selector sequence interaction and the frequency of exon 6 inclusion. However, it is likely that the frequency of exon 6 inclusion is not only determined by the strength of the docking siteselector sequence interaction, but also other factors including the distance between the docking site and each selector sequence, the elongation rate of RNA polymerase along the gene, the strength of the splice sites for each exon 6 variant, RNA binding proteins that enhance or repress splicing of each exon 6 variant, and the potential existence of other RNA structures that would alter the actual length and configuration of the exon 6 cluster pre-mRNA. Thus, it is not surprising that a simple comparison of the strength of the docking site-selector sequence interactions and the frequency of exon 6 use did not reveal a correlation. However, we reasoned that changes in the strength of the base-pairing between the selector sequences and the docking site would change only one of these variables, and therefore allow us to test whether the strength of the docking site-selector sequence interactions plays a role in determining the frequency of exon 6 inclusion.

To test this hypothesis, we first compared the changes in free energy of base-pairing caused by the $\Delta$ Dock, $\Delta$ Left, and $\Delta$ Right mutations for each docking site-selector interaction $(\Delta \Delta G)$ with the fold change in exon 6 inclusion, in the docking site mutation experiments. Specifically, we calculated the $\Delta G$ of each selector sequence with either the WT docking site, or the sequences that remained at the docking site in the $\Delta$ Dock, $\Delta$ Left, and $\Delta$ Right constructs and then calculated the $\Delta \Delta G$ by subtracting the $\Delta G$ in the mutant construct from that in the WT construct. We then compared these values to the fold change observed for each exon between the WT and mutant constructs. As shown in Figure $4 \mathrm{C}$, there were strong correlations between $\Delta \Delta G$ and the fold change in exon 6 inclusion for each docking site deletion construct compared to the WT construct (Pearson's $r$ ranged from -0.38 to -0.65 ).

There were many examples where the observed change in splicing could be explained by the potential base-pairing interactions between the selector sequences and the mutated docking sites. For example, in the $\Delta$ Dock construct, exons $6.1,6.45$, and 6.46 are more frequently included than in the WT construct, and the $\Delta G$ of the selector sequences for each of these exons decreased by no more than $3 \mathrm{kcal} /$ mol, while the remaining selector sequences had an average $\Delta \Delta G$ of $16.0 \mathrm{kcal} / \mathrm{mol}$. Similarly, exons $6.1,6.22-6.24$, and 6.43-6.46 are used more frequently in the $\Delta$ Left construct than in the WT construct (Fig. 4A,B) and again the average 
A

B
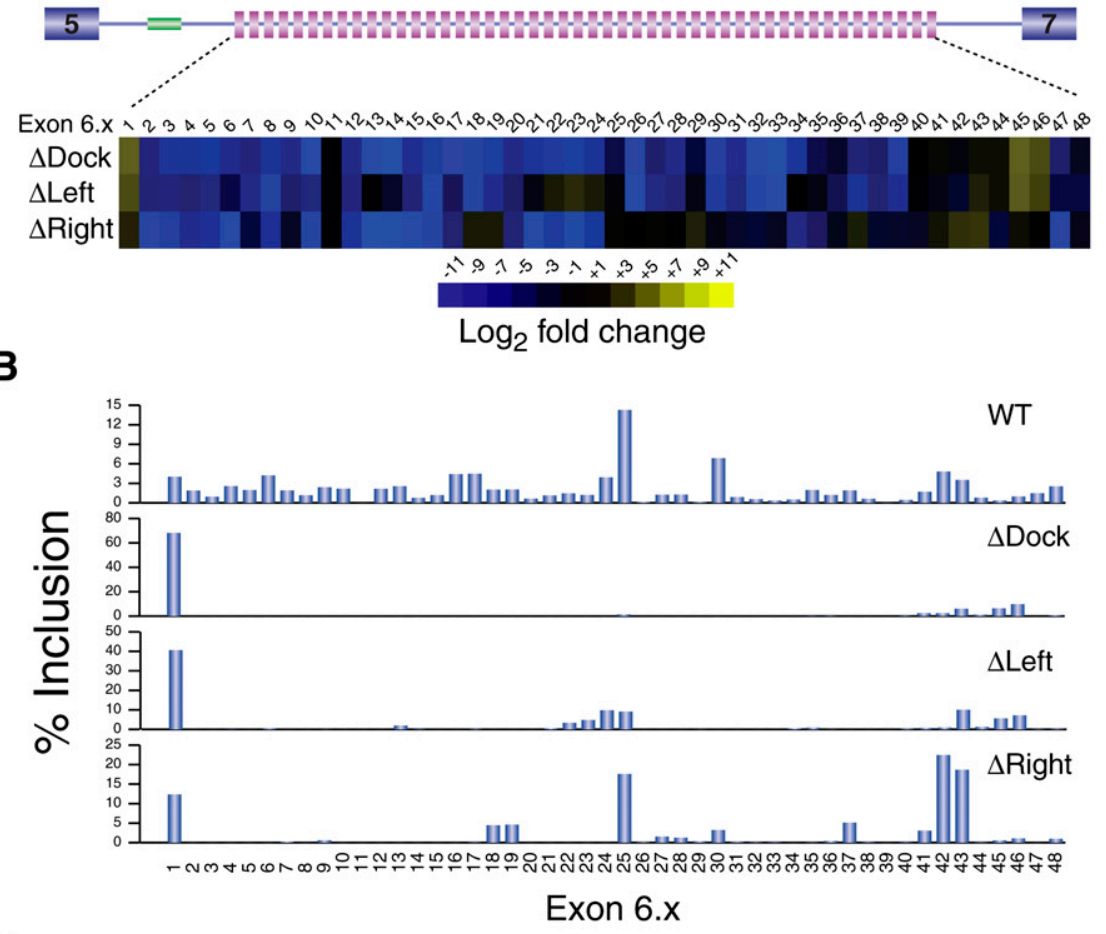

C

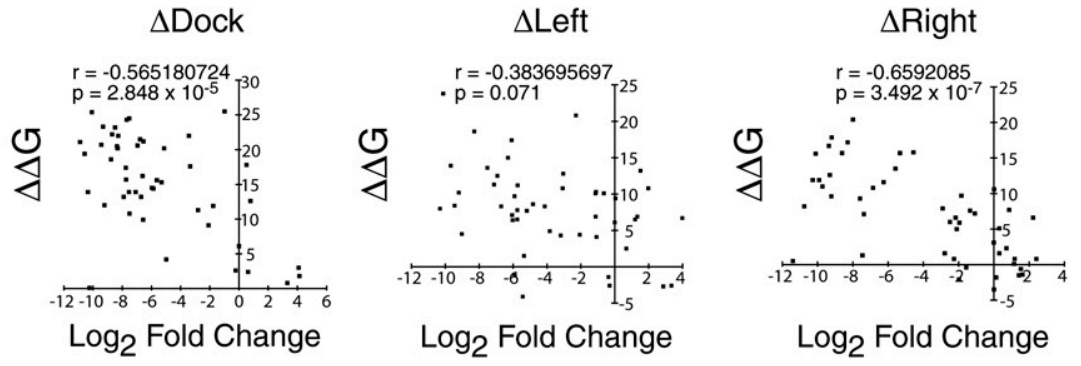

FIGURE 4. Docking site mutations impact the relative frequency of exon 6 inclusion. The mutant docking site constructs were transfected into S2 cells in triplicate and the RT-PCR products were excised and sequenced using the multiplex sequencing method. $(A)$ The $\log _{2}$ fold change of each mutant construct compared to WT Dscam ${ }^{5 / 7 v i r}$ BAC is represented as a heat map. (B) Percent inclusion is plotted for each exon 6 variant. $(C)$ The change in the strength of the predicted docking site-selector sequence interactions $(\Delta \Delta G)$ are plotted as a function of the $\log _{2}$ fold change in the frequency of inclusion for each docking site deletion construct tested.

$\Delta \Delta G$ for these exons is less than for exons that are not more frequently included $(\Delta \Delta G$ of 5.5 vs. $8.5 \mathrm{kcal} / \mathrm{mol})$. In the $\Delta$ Right construct, exons $6.1,6.18,6.19,6.29,6.37,6.42$, and 6.43 have a $\log _{2}$ fold change of at least 1.0 compared to the WT construct and have an average $\Delta \Delta G$ of $0.69 \mathrm{kcal} / \mathrm{mol}$, while the remaining exons have an average $\Delta \Delta G$ of 8.18 $\mathrm{kcal} / \mathrm{mol}$. Thus, for each construct, the change in the frequency of exon inclusion is strongly correlated with the change in the strength of the interaction between the docking site and the selector sequence. These observations are consistent with the hypothesis that the strength of the basepairing interactions is an important determinant of the frequency of exon 6 inclusion.
To more directly test this hypothesis, we identified five selector sequences that were associated with either frequent exon 6 inclusion (exons 6.17 [3.87\% of Dscam ${ }^{5 / 7 v i r}$ BAC transcripts], 6.25 [13.84\%], and $6.30[6.86 \%])$ or infrequent exon 6 inclusion (exons 6.08 $[1.05 \%]$ and $6.45[0.03 \%])$. The "strong" selector sequences of the three frequently included exons were replaced with the "weak" selector sequences from the two infrequently included exons. Conversely, the weak selector sequences of the weakly included exons were replaced with the strong selector sequences from the frequently included exons. We then transfected these BACs into S2 cells and assayed the frequency of exon 6 inclusion with multiplex high-throughput sequencing as above. The results show that when the strong selector sequences were changed to weak selector sequences, the frequency of these exons decreased (Fig. 5A). Conversely, when the weak selector sequences were changed to strong selector sequences, the frequency of these exons increased.

Interestingly, changing selector sequences to either a strong or a weak selector sequence had consistent effects across all of the mutants. For example, when the weak selector sequences were mutated to the 6.30 selector sequence, this resulted in the greatest increase in the usage of both exons 6.08 and 6.45 compared to the other "weak to strong" mutations. Also, mutating the 6.08 and 6.45 selector sequences to the 6.17 selector sequence resulted in the smallest change in exon 6.08 and 6.45 usage. The same trend was seen for the "strong to weak" selector sequence mutations. Mutating the strong selector sequences to the 6.45 selector sequence resulted in a greater decrease in the downstream exon 6 variant compared to mutating the strong selector sequences to the 6.08 sequences. This effect was true for all three strong to weak mutants.

To more quantitatively assess the effect of changing the strength of the docking site-selector sequence interactions on the frequency of exon 6 inclusion, we compared the change in $\Delta G$ of each of the predicted docking site-selector sequence interaction $(\Delta \Delta G)$, with the fold change in exon 6 inclusion. This analysis again revealed a striking correlation between $\Delta \Delta G$ and the change in exon 6 inclusion frequency (Fig. 5B). In fact, Pearson's $r$ is -0.57 for all data points 
A

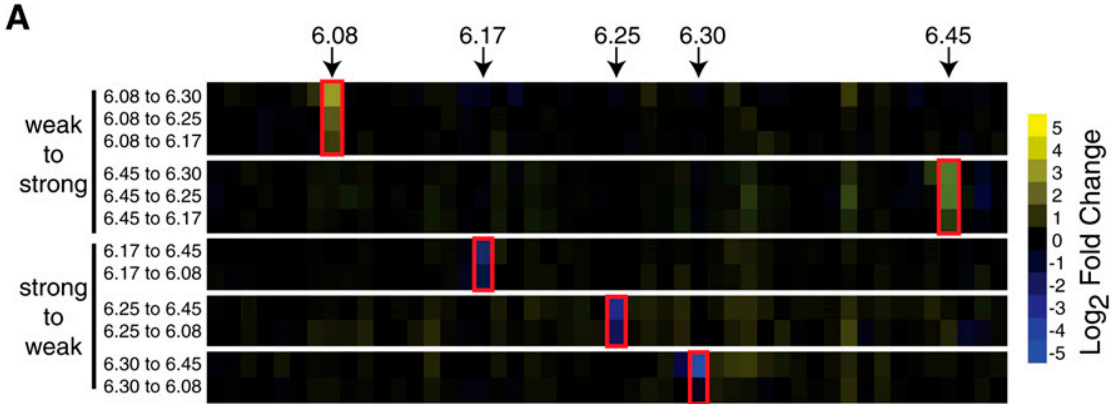

B

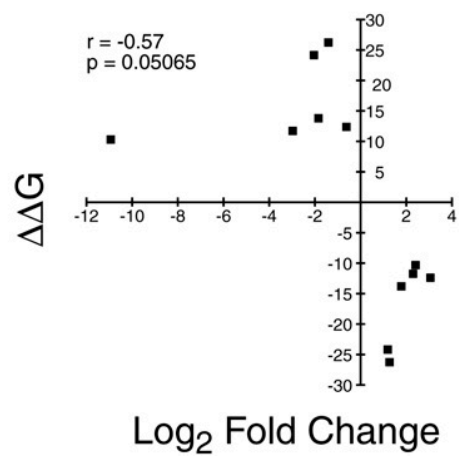

FIGURE 5. Selector sequences determine the efficiency of exon 6 inclusion. (A) The selector sequences of individual exons were replaced in the Dscam ${ }^{5 / 7 v i r}$ BAC as indicated on the left. Transfection and multiplex sequencing were used to determine the fold change of exon 6 inclusion with respect to the unmodified BAC. The fold change $\left(\log _{2}\right)$ was plotted as a heat map. In each experiment the exon containing the modified selector sequence is highlighted in red. $(B)$ The change in the strength of the predicted docking site-selector sequence interaction $(\Delta \Delta G)$ is plotted as a function of the fold change in the frequency of inclusion of the modified exon 6 variant.

$(P=0.05065)$ and $-0.817919355(P=0.002096)$ when removing the outlier data point of changing the 6.30 selector sequence to that of 6.45 (for which the percent inclusion is extremely low and likely to be somewhat inaccurate). The striking correlation between the strength of the docking siteselector sequence interactions and inclusion frequency observed when either the docking site or selector sequences are manipulated strongly suggests that in the endogenous location, the strength of the interaction between the docking site and selector sequence plays a role in determining the relative exon 6 inclusion frequency.

\section{DISCUSSION}

Though a role of RNA secondary structures in alternative splicing has been known for years (Xing and Lee 2006), the function of such structural elements is much less studied than that of proteins. In this study, we examined the function of conserved sequence elements (the docking site and selector sequences), which have been proposed, but not shown, to play a role in Dscam mutually exclusive splicing (Graveley 2005; Anastassiou et al. 2006). Our data reveal that the docking site is indeed functionally involved in mutually exclusive splicing as it is required for efficient exon
6 inclusion. We also show that this function of the docking site is evolutionarily conserved, supporting the hypothesis that these conserved elements are under strong purifying selection. We also showed that the strength of the docking site-selector sequence interaction plays a role in determining the inclusion frequency of the exon 6 variants. Thus, this work demonstrates that conserved sequence elements in introns that form RNA secondary structures play a functional role in mutually exclusive splicing.

One interesting finding we made is that the inclusion frequency of the exon 6 variants cannot be predicted merely by calculating the strength of the docking site-selector sequence base-pairing interactions across the entire exon 6 cluster. However, the converse was true when we manipulated these interactions by either deleting the docking site or moving individual selector sequences. In these situations, there was a strong correlation between the strength of the docking siteselector sequence interactions and the frequency of exon 6 inclusion. When selector sequences of infrequently included exon 6 variants were changed to selector sequences of frequently included exons, the inclusion frequency of the downstream exon 6 variant increased. When the counter experiment was performed, the inclusion frequency of the downstream exon 6 variant decreased. Thus, selector sequence identity has a strong effect on exon 6 inclusion frequencies.

However, the fact that this correlation can only be observed when the strength of the interactions is perturbed indicates that the frequency of exon 6 inclusion cannot be fully explained by the selector sequence alone. Rather, other factors such as the position of the exon within the cluster, the strength of the splice sites of each exon 6 variant, other RNA structures, the rate of transcription, and RNA binding proteins likely play important roles in determining the frequency of exon 6 inclusion. In fact, it is likely that the docking site and selector sequences are part of a larger integrative system that functions to facilitate mutually exclusive splicing in Dscam. For example, the RNA binding protein HRP36 has been shown to bind throughout the Dscam exon 6 cluster and is necessary for exon 6 mutually exclusive splicing fidelity (Olson et al. 2007). Further work will be necessary to elucidate these other aspects of the exon 6 mutually exclusive splicing mechanism and to determine whether or not these mechanisms are limited to the Dscam exon 6 cluster or the Dscam gene, or operate in other genes and eukaryotic organisms. 


\section{MATERIALS AND METHODS}

\section{Dscam mutant constructs}

A 102-kbp P[acman] (Venken and Bellen 2005; Venken et al. 2006,Venken et al 2009) BAC containing the D. melanogaster Dscam gene was obtained from the laboratory of Hugo Bellen. Recombineering (Warming et al. 2005) was performed in E. coli strain SW102 (Biological Resources Branch of the National Cancer Institute: http:// web.ncifcrf.gov/research/brb/recombineeringInformation.aspx), with the following modifications. To insert the galK cassette into the targeted region of the Dscam BAC, the galK cassette and 200-500bp homologous arms were cloned into the vector PCRII-TOPO (Invitrogen). The galK cassettes with homologous arms were then PCR amplified, digested with $D p n I$ at $37^{\circ} \mathrm{C}$ for $1 \mathrm{~h}$ to remove the vector template, and recombined into the Dscam BAC. For the counter selections, mutations of interest were cloned into the PCRIITOPO vector, PCR amplified, and recombined into Dscam BAC clones containing the galK targeting cassette. In order to differentiate transcripts from the BAC and endogenous Dscam gene, sequences in constitutive exons 5 and 7 in each mutant Dscam BAC construct were mutated to the corresponding sequences from $D$. virilis. All mutations were verified by sequencing. Construction of the Dscam BAC clones are described in the Supplemental Material.

\section{Daphnia pulex minigene transfection assay}

Daphnia pulex was obtained from Carolina Biologicals. Genomic DNA was harvested and the 7000-bp Dscam exon 6 cluster was amplified in two halves with LaTaq (Takara) according to the manufacturer's instructions. The PCR products were cloned into the pMT/V5 TOPO vector (Invitrogen) and later joined into one clone containing the entire 7-kb exon 6 cluster. The $D$. pulex minigene and a D. pulex $\Delta$ Dock construct were transfected into D. melanogaster S2 cells as follows. Cells were plated in a six-well tissue culture dish at a density of $1 \times 10^{6}$ cells per well and allowed to adhere. The media was removed and the cells were transfected using $1 \mu \mathrm{g}$ of DNA and $6 \mu \mathrm{L}$ of Cellfectin reagent. The transfection mixture was removed after $5 \mathrm{~h}$, replaced with fresh media, and incubated for $24 \mathrm{~h}$ at $27^{\circ} \mathrm{C}$. Expression of the pMT/V5 vector was induced with $15 \mu \mathrm{L}$ of 100 $\mu \mathrm{M} \mathrm{CuSO}_{4}$ and incubated for $24 \mathrm{~h}$ at $27^{\circ} \mathrm{C}$. Total RNA was harvested using Trizol (Invitrogen) according to the manufacturer's protocol. Details on the construction of the D. pulex mini gene and on a $D$. pulex construct where the docking site was deleted, RT-PCR, and deep sequencing methods can be found in the Supplemental Material.

\section{Transfection of Dscam BAC constructs}

BAC DNA was prepared using the Large Construct Kit (QIAGEN) as per the manufacturer's instructions. D. melanogaster S2 cells in Express Five medium (Invitrogen) were plated in a six-well culture dish at a cell density of $8 \times 10^{5}$ cells per well. Cells were allowed to adhere to the plate for $\sim 30 \mathrm{~min}$. The media was removed and the cells were transfected in triplicate wells with $4 \mu \mathrm{g}$ of BAC DNA, $8 \mu \mathrm{L}$ of Cellfectin (Invitrogen), and SFM up to a total volume of $800 \mu \mathrm{L}$ per well. The transfection mixture was removed after $4 \mathrm{~h}$ and replaced with $2 \mathrm{~mL}$ of SFM supplemented with L-glutamine. The S2 cells were incubated for $48 \mathrm{~h}$ at $27^{\circ} \mathrm{C}$. Total RNA was isolated from each well with Trizol (Invitrogen) according to the manufacturer's instructions.

\section{Measuring Dscam exon 6 skipping}

The degree to which the Dscam BAC docking site mutations affected exon 6 skipping, i.e., exon 5 spliced to exon 7, was measured using the $\Delta \Delta$ CT quantitative PCR method (Livak and Schmittgen 2001). Total RNA from the transfections was reverse transcribed and amplified using the One Step qRT-PCR kit (Invitrogen) or the Qiagen one-step RT-PCR kit (QIAGEN) as per the manufacturer's instructions. Approximately $250 \mathrm{ng}$ of total RNA was added to a 25 $\mu \mathrm{L}$ reaction and reverse transcribed at $50^{\circ} \mathrm{C}$ for $30 \mathrm{~min}$, followed by 45 cycles of PCR with an annealing and extension temperature of $60^{\circ} \mathrm{C}$. The skipped splicing product, where exon 5 is spliced to exon 7, was amplified using primers Exon5-7JunctionF [5'TGGT CATCACAGATGGTACAAGTT3' ${ }^{\prime}$, which anneals to the 5-7 junction, and Dvir-X7spec-Rev [5'AGCCTCGGCGGATTCCTGATCG TTGCGC3'], which anneals to the BAC exon 7 transcript. A 421bp product was detected using a final concentration of $200 \mathrm{nM}$ of probe (EX7probe [5' - 6-carboxyfluorescein-TTCTTCACGGATTC GATGCG-BHQ1-3']). To control for the total amount of Dscam BAC RNA in the samples, a 474-bp calibrator product where Dscam exon 7 is spliced to exon 8 was also amplified using the primers D.vir.X7virF [5'GCGCAACGATCAGGAATCCGCCGAG GCT3'] and EX7-8junctionR [5'GCACGGTTATCTCGCTCCCA3']. The probe EX7probe2 [5'-6-carboxyfluorescein-CCTTCCAGGAA GAGACCATG-BHQ1-3'], at a final concentration of $200 \mathrm{nM}$, was used to detect the amplified product. No amplification was detected with the primer and probe sets when tested with template RNA from untransfected S2 cells, indicating that the primers and probes do not amplify and detect endogenous RNA.

\section{RT-PCR}

Reverse transcription followed by PCR was performed on each RNA sample using the Superscript III One-Step RT-PCR System with Platinum Taq DNA Polymerase kit (Invitrogen). Five hundred ng of total RNA was added to a $25 \mu \mathrm{L}$ reaction and reverse transcribed at $50^{\circ} \mathrm{C}$ for $30 \mathrm{~min}$, followed by 35 cycles of PCR with an annealing temperature of $67^{\circ} \mathrm{C}$. The primers DvirX5spec-Fwd [5'GCATCGTTTGACTGGAGAGACGCGTCTG3'] and Dvir-X7spec-Rev [5'AGCCTCGGCGGATTCCTGATCGTTG CGC3'] were used to amplify Dscam exon 6 BAC products, while the primers D.mel.X5F [5'ACATCGTCTGACCGGAGAAACCC GATTA3'] and D.mel.X7R [5'CGCCTCCGCCGATTCCTGGTCG TTTCTT3'] were used to amplify the endogenous exon 6 products. Products were electrophoresed in a $2 \%$ agarose gel and an $\sim 560$ bp product containing the spliced exon 6 variants were excised and gel purified using the QIAquick Gel Extraction Kit (QIAGEN). To assay for the total amount of Dscam BAC RNA in the samples, a 474 bp Dscam exon 7 and 8 product was amplified using the primers D.vir.X7virF and EX7-8junctionR.

\section{Exon 6 utilization assay}

Relative exon 6 use was assayed using multiplex high-throughput sequencing. Sequencing libraries were prepared as follows. Ten to $30 \mathrm{ng}$ of each BAC and endogenous RT-PCR product containing exons 5, 6, and 7, was amplified using Phusion DNA polymerase (Finnzymes) (25 cycles; $25 \mu \mathrm{L}$; annealing temperature $60^{\circ} \mathrm{C}$ ). Primers SEQ.VIR.X5VIRF [5'CAAGCAGAAGACGGCATACGA GCATCGTTTGACTGGAGAGACGCGTCTG3'] and SEQ.MEL.X5 
VIRF [5'CAAGCAGAAGACGGCATACGAACATCGTCTGACC GGAGAAACCCGATTA3'] anneal to exon 5 in the RT-PCR product and contain sequences necessary for cluster generation on Illumina flow cells (in bold). The reverse primer [5'AATGATACGG CGACCACCGACAGGTTCAGAGTTCTACAGTCCGACGATC ${ }^{\star * * A T}$ GAACTTGTACCAT3'] anneals to constitutive exon 7, contains sequence for cluster generation on Illumina flow cells (in bold), a sequence for the Illumina small RNA sequencing primer to anneal (in italics), and a 3 -ncleotide barcode noted as ${ }^{* * *}$. PCR products were electrophoresed in a $2 \%$ agarose gel and an $\sim 280$-bp product was excised and gel purified using the QIAquick Gel Extraction Kit (QIAGEN). Pools of 22 samples were loaded into six lanes on an Illumina Genome Analyzer IIx (GAIIx) and subjected to 40 cycles of Illumina sequencing.

\section{Thermodynamic calculations}

The $\Delta G$ of each docking site-selector sequence interaction was calculated using UNAFold on the DINAMelt Server (http://dinamelt. bioinfo.rpi.edu/twostate.php) (Markham and Zuker 2005, 2008).

\section{SUPPLEMENTAL MATERIAL}

Supplemental material can be found at http://www.rnajournal.org.

\section{ACKNOWLEDGMENTS}

We thank Hugo Bellen and Koen Venken for the Dscam BAC, members of the Graveley lab for discussions, and the UCHC Translational Genomics Core for use of the Illumina GAIIx. This work was supported by a grant from the NIH (2R01GM067842-09) to B.R.G.

Authors' contributions: G.E.M., S.O., and C.J.M. performed the experiments; G.E.M., S.O., C.J.M and B.R.G. designed the experiments; G.E.M, S.O., C.J.M., and B.R.G. analyzed the data; G.E.M. wrote the paper with input from all the authors.

Received November 2, 2010; accepted November 16, 2010.

\section{REFERENCES}

Anastassiou D, Liu H, Varadan V. 2006. Variable window binding for mutually exclusive alternative splicing. Genome Biol 7: R2. doi: 10.1186/gb-2006-7-1-r2.

Brites D, McTaggart S, Morris K, Anderson J, Thomas K, Colson I, Fabbro T, Little TJ, Ebert D, Du Pasquier L. 2008. The Dscam homologue of the crustacean Daphnia is diversified by alternative splicing like in insects. Mol Biol Evol: 25: 1429-1439.

Chen M, Manley J. 2009. Mechanisms of alternative splicing regulation: insights from molecular and genomics approaches. Nat Rev Mol Cell Biol 10: 741-754.
Copeland NG, Jenkins NA, Court DL. 2001. Recombineering: a powerful new tool for mouse functional genomics. Nat Rev Genet 2: 769779.

Fededa JP, Petrillo E, Gelfand MS, Neverov AD, Kadener S, Nogues G, Pelisch F, Baralle FE, Muro AF, Kornblihtt AR. 2005. A polar mechanism coordinates different regions of alternative splicing within a single gene. Mol Cell 19: 393-404.

Graveley BR. 2005. Mutually exclusive splicing of the insect Dscam pre-mRNA directed by competing intronic RNA secondary structures. Cell 123: 65-73.

Grover A, Houlden H, Baker M, Adamson J, Lewis J, Prihar G, Pickering-Brown S, Duff K, Hutton M. 1999. 5' Splice site mutations in tau associated with the inherited dementia FTDP17 affect a stem-loop structure that regulates alternative splicing of exon 10. J Biol Chem 274: 15134-15143.

Kornblihtt AR. 2007. Coupling transcription and alternative splicing. Adv Exp Med Biol 623: 175-189.

Lee C, Kim N, Roy M, Graveley BR. 2010. Massive expansions of Dscam splicing diversity via staggered homologous recombination during arthropod evolution. RNA 16: 91-105.

Livak KJ, Schmittgen TD. 2001. Analysis of relative gene expression data using real-time quantitative PCR and the $2^{-\Delta \Delta C}$ method. Methods 25: 402-408.

Markham NR, Zuker M. 2005. DINAMelt web server for nucleic acid melting prediction. Nucleic Acids Res 33: W577-W581.

Markham NR, Zuker M. 2008. UNAFold: software for nucleic acid folding and hybridization. Methods Mol Biol 453: 3-31.

Nilsen TW, Graveley BR. 2010. Expansion of the eukaryotic proteome by alternative splicing. Nature 463: 457-463.

Olson S, Ni JF, Graveley BR. 2010. Global analysis of Dscam expression in D. melanogaster. RNA (in press).

Pan Q, Shai O, Lee LJ, Frey BJ, Blencowe BJ. 2008. Deep surveying of alternative splicing complexity in the human transcriptome by high-throughput sequencing. Nat Genet 40: 1413-1415.

Schmucker D, Clemens JC, Shu H, Worby CA, Xiao J, Muda M, Dixon JE, Zipursky SL. 2000. Drosophila Dscam is an axon guidance receptor exhibiting extraordinary molecular diversity. Cell 101: 671-684.

Venken KJ, Bellen HJ. 2005. Emerging technologies for gene manipulation in Drosophila melanogaster. Nat Rev Genet 6: 167178.

Venken KJ, He Y, Hoskins RA, Bellen HJ. 2006. P[acman]: a BAC transgenic platform for targeted insertion of large DNA fragments in D. melanogaster. Science 314: 1747-1751.

Venken KJ, Carlson JW, Schulze KL, Pan H, He Y, Spokony R, Wan $\mathrm{KH}$, Koriabine M, de Jong PJ, White KP, et al. 2009. Versatile $\mathrm{P}$ [acman] BAC libraries for transgenesis studies in Drosophila melanogaster. Nat Methods 6: 431-434.

Wang ET, Sandberg R, Luo S, Khrebtukova I, Zhang L, Mayr C, Kingsmore SF, Schroth GP, Burge CB. 2008. Alternative isoform regulation in human tissue transcriptomes. Nature 456: 470476.

Warming S, Costantino N, Court DL, Jenkins NA, Copeland NG. 2005. Simple and highly efficient BAC recombineering using galK selection. Nucleic Acids Res 33: e36. doi: 10.1093/nar/gni035.

Xing Y, Lee C. 2006. Alternative splicing and RNA selection pressure-evolutionary consequences for eukaryotic genomes. Nat Rev Genet 7: 499-509. 

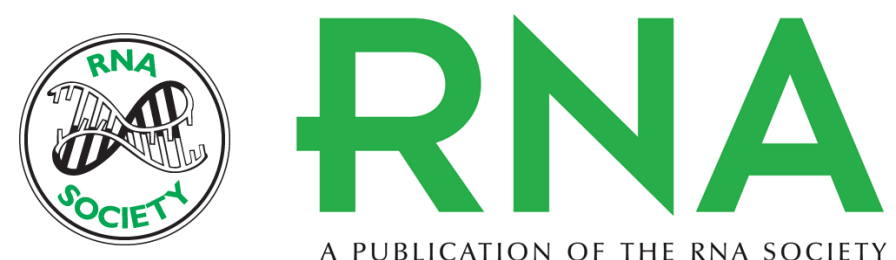

A PUBLICATION OF THE RNA SOCIETY

\section{Competing RNA secondary structures are required for mutually exclusive splicing of the Dscam exon 6 cluster}

Gemma E. May, Sara Olson, C. Joel McManus, et al.

RNA 2011 17: 222-229 originally published online December 15, 2010

Access the most recent version at doi:10.1261/rna.2521311

\section{Supplemental http://rnajournal.cshlp.org/content/suppl/2010/11/24/rna.2521311.DC1 \\ Material}

References This article cites 21 articles, 3 of which can be accessed free at: http://rnajournal.cshlp.org/content/17/2/222.full.html\#ref-list-1

\section{License}

Email Alerting Receive free email alerts when new articles cite this article - sign up in the box at the Service top right corner of the article or click here.

\section{|||||||| Providing Precise Solutions for your research.}

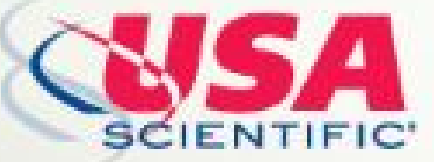

To subscribe to $R N A$ go to:

http://rnajournal.cshlp.org/subscriptions 